\title{
Antitumor and antimetastatic activities of a novel benzothiazole- 2-thiol derivative in a murine model of breast cancer
}

\author{
Xiaolin $\mathrm{Hu}^{1, *}$, Sen $\mathrm{Li}^{2, *}$, Yan $\mathrm{He}^{3, *}$, Ping $\mathrm{Ai}^{3, *}$, Shaoyong $\mathrm{Wu}^{3, *}$, Yonglin $\mathrm{Su}^{4, *}$, Xiaolin \\ $\mathbf{L i}^{5}$, Lei Cai ${ }^{6}$, Xingchen Peng ${ }^{3}$ \\ ${ }^{1}$ Department of Nursing, West China Hospital, Sichuan University, Chengdu, China \\ ${ }^{2}$ Department of Spinal Surgery, Traditional Chinese Medicine Hospital of SouthWest Medical University, Luzhou, China \\ ${ }^{3}$ Department of Medical Oncology, Cancer Center, State Key Laboratory of Biotherapy, West China Hospital, Sichuan \\ University, Chengdu, China \\ ${ }^{4}$ Department of Rehabilitation, West China Hospital, Sichuan University, Chengdu, China \\ ${ }^{5}$ Department of Pathophysiology, Basic Medical College, Jilin University, Changchun, China \\ ${ }^{6}$ Hepatobiliary Surgery Institute, Southwest Hospital, Third Military Medical University, Chongqing, China \\ *These authors have contributed equally to this work
}

Correspondence to: Xingchen Peng, email: pxx2014@sohu.com

Keywords: benzothiazole-2-thiol derivative, breast cancer, proliferation, metastasis, apoptosis

Received: November 24, $2016 \quad$ Accepted: December 21, $2016 \quad$ Published: January 02, 2017

\section{ABSTRACT}

The prognosis of metastatic breast cancer is always very poor. Thus, it is urgent to develop novel drugs with less toxicity against metastatic breast cancer. A new drug (XC-591) derived from benzothiazole-2-thiol was designed and synthesized in our lab. In this study, we tried to assess effects of XC-591 treatment on primary breast cancer and pulmonary metastasis in 4T1 mice model. Furthermore, we tried to discover its possible molecular mechanism of action. MTT experiment showed XC-591 had significant anti-cancer activity on diverse cancer cells. Furthermore, XC-591 significantly suppressed the proliferation of 4T1 cells by colony formation assay. The in vivo results displayed that XC-591 could inhibit the growth and metastasis in 4T1 model. Moreover, histological analysis revealed that XC-591 treatment increased apoptosis, inhibited proliferation and angiogenesis in vivo. In addition, XC-591 did not contribute to obvious drug associated toxicity during the whole study. Molecular mechanism showed XC-591 could inhibit RhoGDI, activate caspase-3 and decrease phosphorylated Akt. The present data may be important to further explore this kind of new small-molecule inhibitor.

\section{INTRODUCTION}

Breast cancer incidence and mortality have been increasing in China. It has been estimated that 268,600 Chinese women developed breast cancer and 69,500 died of breast cancer in the year 2015 [1]. Therapeutic options such as chemotherapy, radiotherapy, hormonal therapy, and targeted therapies are often used to treat patients with breast cancer [2]. In the last decades, early detection and proper treatment have helped reduce breast cancer mortality. However, distant metastases are still the main cause of breast cancer associated deaths [3]. Thus, it is urgent to develop novel drugs with less toxicity against metastatic breast cancer. Considering safety and increasing anti-cancer efficacy, smallmolecule inhibitors are being deeply investigated as a new treatment method.

Benzothiazole derivatives have diverse biological functions, including anti-inflammatory, anticonvulsant, antimicrobial and antitumor activities $[4,5]$. But previous studies used 2-aminobenzothiazoles or 2-arylbenzothiazoles to design benzothiazole derivatives. In our lab, we used the benzothiazole-2-thiol as a functional group to develop a new series of compounds. Among these small-molecule compounds, XC-591 had obvious anti-tumor activity on diverse cancer cells in vitro, which was more effective than cisplatin (a platinum based chemotherapeutic drug) [6]. However, the exact anti-cancer effects of XC-591 in vivo have not been reported. In this study, we attempted to 
investigate the effect of XC-591 on suppressing both murine breast cancer and pulmonary metastases. In addition, possible molecular mechanism of action was also studied.

\section{RESULTS}

\section{RhoGDI was increased in cancer cells}

Western blot was conducted to check RhoGDI expression in cancer cell lines (including 4T1 and A549) and normal cell lines (including MM3MG and HK2). RhoGDI was obviously increased in two cancer cell lines, when compared with two normal cells (Figure 1A).

\section{The cytotoxicity effect of XC-591 on tumor cells}

RhoGDI specific inhibitor XC-591 was synthesized in our lab and the chemical structure was shown in Figure 1B. As shown in Table 1, XC-591 inhibited proliferation of many cancer cells (including CT26, 4T1, LLC, B16, MethA, A549, HeLa and DU145). Howerver, no apparently toxicity on normal cells (including MM3MG, HK2 and LO2) was observed (Table 1). Compared with other tumor cells, 4T1 was more sensitive to XC-591 than others and thus was selected for further study.

\section{XC-591 suppressed proliferation of 4T1}

Colony formation assay demonstrated that there was a marked decrease in the colony number from $364 \pm 46.4$ in the untreated $4 \mathrm{~T} 1$ cells to $189 \pm 35.2$ and $56 \pm 12$, respectively, in 1.25 and $2.5 \mu \mathrm{M} \mathrm{XC}-591$ treated tumor cells $(\mathrm{P}<0.05)$ (Figure 2). These results showed that the proliferative activity of $4 \mathrm{~T} 1$ cells was inhibited by XC-591 in a dose-dependent way.

\section{Anti-tumor effect of XC-591 in vivo}

As shown in Figure 3A, it is obvious that the treatment with XC-591 resulted in primary tumor growth regression of $48.5 \%$ and $79 \%$, in $50 \mathrm{mg} / \mathrm{kg} \mathrm{XC}-591$ treated group and $100 \mathrm{mg} / \mathrm{kg}$ XC-591 treated group respectively, when compared with the untreated group. The similar

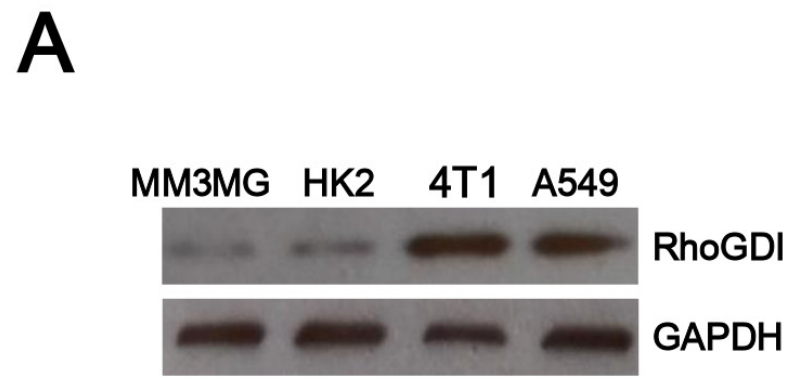

results about tumor weight were also observed. We found the average weight of the tumors was decreased by $46.9 \%$ and $71.8 \%$ in $50 \mathrm{mg} / \mathrm{kg} \mathrm{XC-591} \mathrm{treated} \mathrm{group} \mathrm{and} 100 \mathrm{mg} /$ kg XC-591 treated group, respectively, when compared with the untreated control (Figure 3B).

\section{XC-591 inhibited the metastasis of 4T1 from the primary site to lungs in vivo}

As shown in Figure 4 and Table 2, our data showed that the untreated group had a median of 40.5 metastases/lungs (range, 28-56 metastases). However, the median of $50 \mathrm{mg} / \mathrm{kg}$ XC-591 treated group was only 19.5 metastases/lungs (range, 13-27 metastases). Furthermore, mice treated with $100 \mathrm{mg} / \mathrm{kg}$ XC-591 had a median of 6 metastases/lungs (range, $0-8$ metastases). More importantly, 3 mice with no visible lung metastases were observed in the group treated with $100 \mathrm{mg} / \mathrm{kg} \mathrm{XC}$ 591 (Table 2). Thus, XC-591 could inhibit primary tumor growth and distant metastases in 4T1 model.

\section{Inhibition of proliferation (PCNA) and increase of apoptosis (TUNEL)}

Tumor sections of each group were stained with anti-PCNA antibody and TUNEL reagent in order to evaluate proliferation and apoptosis rate. Compared with the control group, XC-591 significantly decreased percentages of PCNA-positive nuclei (Figure 5A\&5B). Meanwhile we could find a large area of necrosis in the sections of both XC-591 treated groups (Figure 5A). Moreover, in a concentration-dependent way, XC-591 increased percentage of TUNEL-positive nuclei, when compared with the untreated group (Figure 5C\&5D). Thus, XC-591 could directly inhibit proliferation and induce apoptosis in vivo.

\section{XC-591 inhibited angiogenesis in vivo}

As shown in Figure 5E\&5F, compared with the untreated group with high MVD, lower MVD could be observed in XC-591 treated groups. Thus, these data showed that XC-591 could inhibit angiogenesis.

B

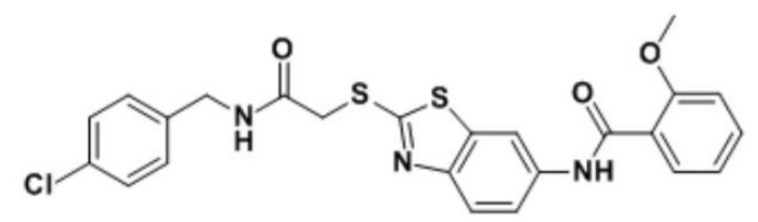

Figure 1: A. RhoGDI expression was increased in cancer cells. B. The chemical structure of XC-591. 
Table 1: The cytotoxicity effect of XC-591 on tumor cells and normal cells

\begin{tabular}{lcc}
\hline Cell line & Cell type & IC50 ( $\mathbf{M M})(\mathbf{m e a n} \pm \mathbf{S . D .})$ \\
\hline CT26 & Mouse colon carcinoma cell line & $4.3 \pm 0.8$ \\
4 T1 & Mouse mammary tumor cell line & $1.2 \pm 0.3$ \\
LLC & Mouse Lewis lung carcinoma cell line & $6.5 \pm 0.4$ \\
B16 & Mouse melanoma cell line & $5.6 \pm 0.9$ \\
MethA & Mouse fibrosarcoma cell line & $12.3 \pm 2.8$ \\
A549 & Human lung carcinoma cell line & $2.4 \pm 0.7$ \\
HeLa & Human cervical cancer cell line & $4.3 \pm 1.6$ \\
DU145 & Human prostate cancer cell line & $1.9 \pm 1.1$ \\
MM3MG & Mouse mammary normal epithelial cell line & $>40$ \\
HK2 & Human normal kidney cell line & $>40$ \\
LO2 & Human normal liver cell line & $>40$
\end{tabular}

Each cell line was treated with XC-591 for 48 h. Cell viability was detected by MTT assay. Data are expressed as the mean \pm S.D. from three independent experiments.

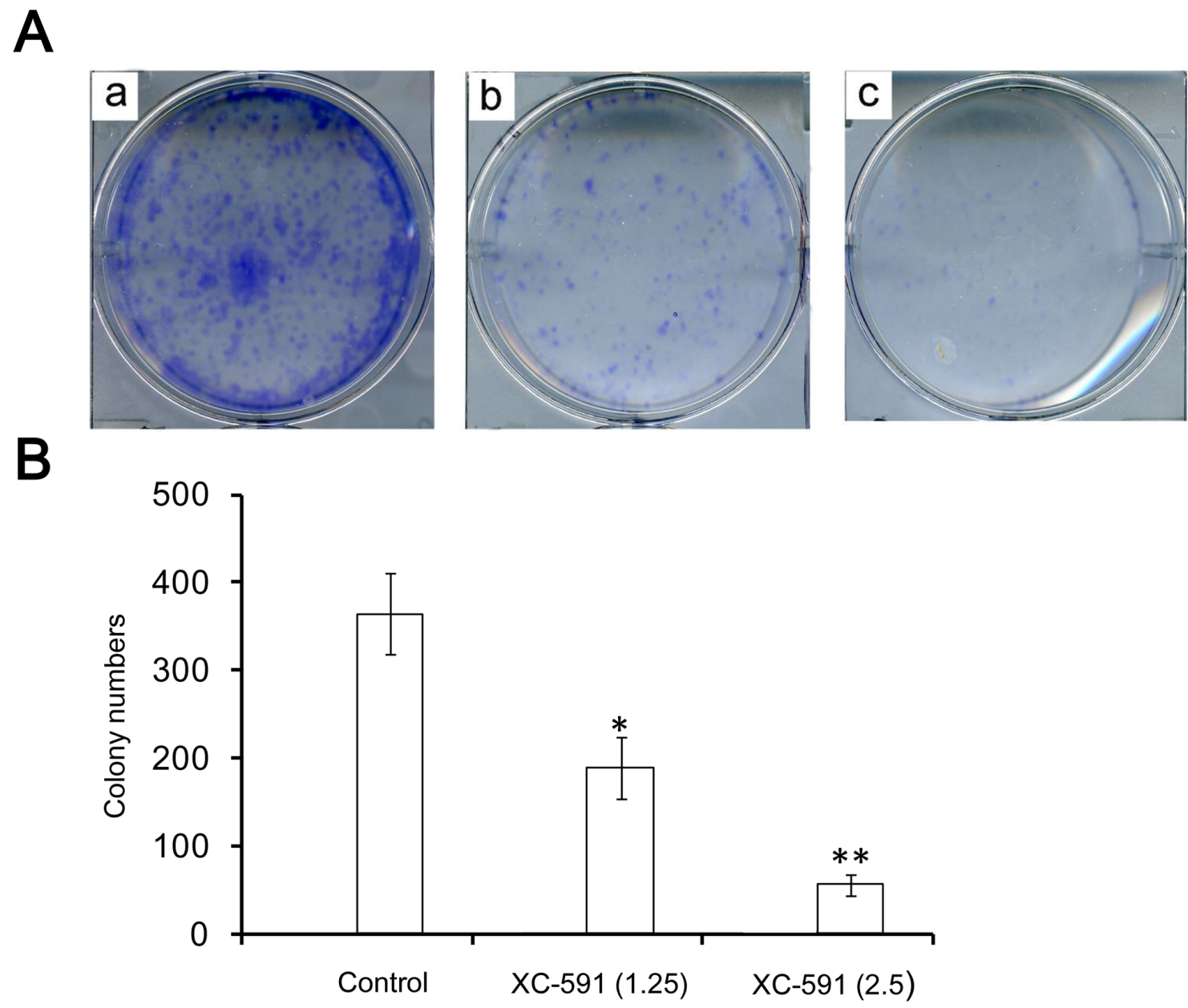

Figure 2: XC-591 could inhibit proliferation of 4T1 in vitro. A. Representative pictures from colony formation assays. a: untreated group; b: $1.25 \mu \mathrm{M} \mathrm{XC}-591$ treated group; c: $2.5 \mu \mathrm{M}$ XC-591 treated group. B. Colony quantification of $4 \mathrm{~T} 1$ cells treated with $0,1.25$ and $2.5 \mu \mathrm{M}$ of $\mathrm{XC}-591$, respectively. * indicates $\mathrm{P}<0.05, * *$ indicates $\mathrm{P}<0.01$. 


\section{Toxicity assessment}

To assess the possible adverse effects of XC-591, weight of mice was monitored every 3 days during the experiment. No weight changes were found after XC591 treatment (Figure 6). In addition, no ruffled fur or toxic death was observed in the XC-591 treated groups. Furthermore, as shown in Table 3, XC-591 treatment did not contribute to blood toxicity and liver toxicity $(\mathrm{P}>0.05$, respectively).

\section{XC-591 inhibited RhoGDI, activated caspase-3 and reduced phosphorylated Akt}

The predicted drug target protein of XC-591 is Rho GDP-dissociation inhibitor 1 (RhoGDI). The protein expression level of RhoGDI was examined by western blot after treatment with XC-591. As shown in Figure 7, RhoGDI was inhibited obviously in the XC-591 treated 4T1 cells.

We investigated whether Akt was involved in XC-591-mediated proliferation inhibitory effect. Our result showed XC-591 clearly decreased phosphorylated Akt (Figure 7). Furthermore, we attempted to study the impact of XC-591 on the activation of caspase-3. XC-591 treatment contributed to an obvious increase of cleaved caspase-3 (Figure 7).

\section{DISCUSSION}

Because an obvious improvement of cancer molecular biology has been achieved recently, novel
A

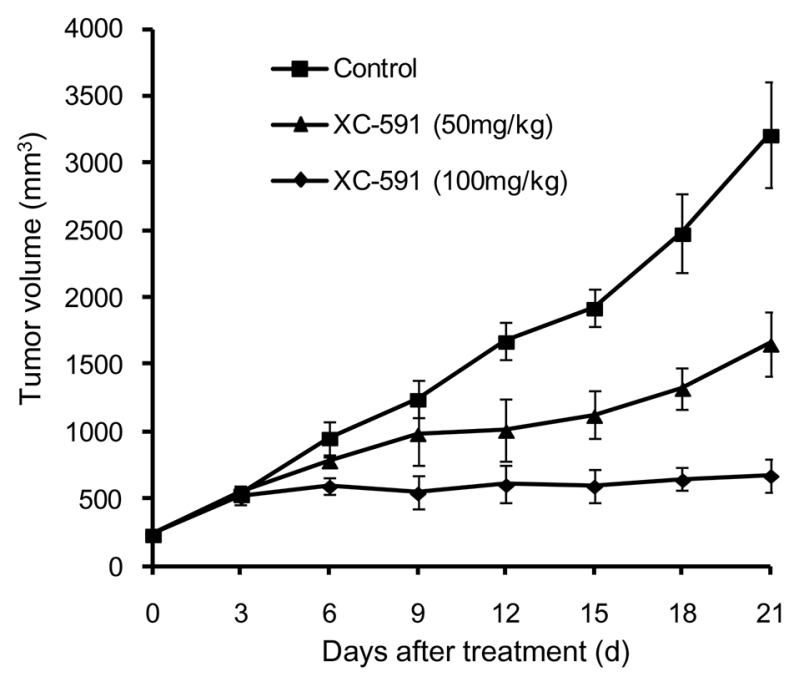

B

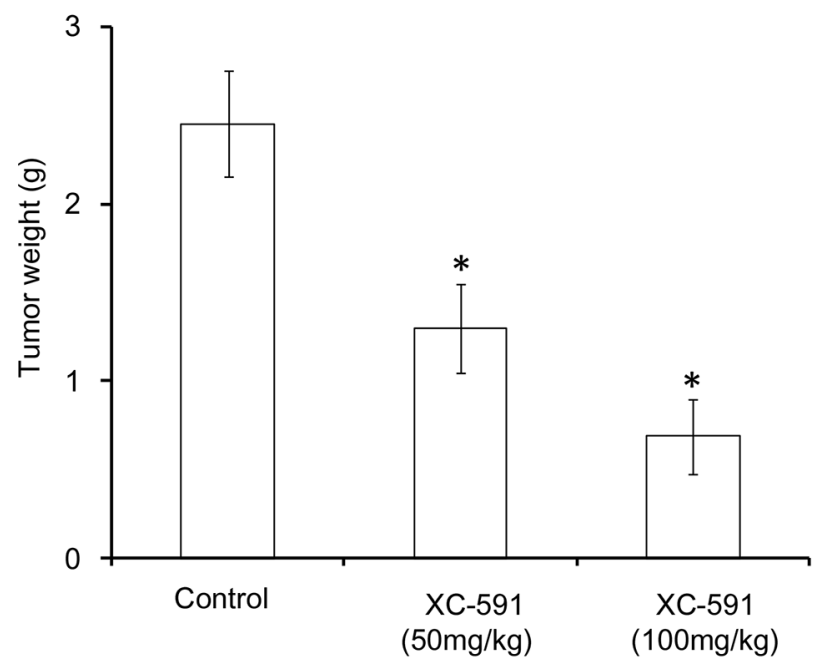

Figure 3: Anti-tumor effect of XC-591 in vivo. A. Tumor growth curves. B. Tumor weight. * indicates $\mathrm{P}<0.05$.
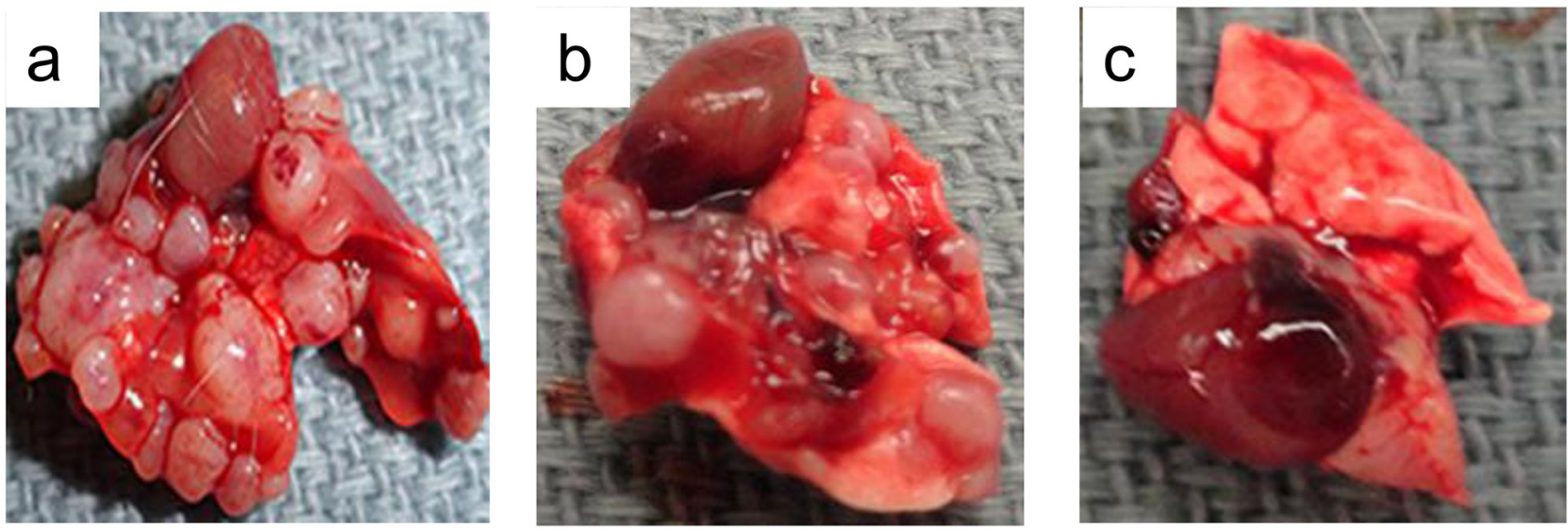

Figure 4: Representative pictures from metastatic lungs in mouse 4T1 model. a: untreated group; b: $50 \mathrm{mg} / \mathrm{kg}$ XC-591 treated group; c: $100 \mathrm{mg} / \mathrm{kg} \mathrm{XC-591} \mathrm{treated} \mathrm{group.}$ 
Table 2: Lung metastatic nodules of each group

\begin{tabular}{lcc}
\hline Groups & Median no. of metastases (range)/lung & \% metastasis-free mice \\
\hline Control & $40.5(28-56)$ & 0 \\
XC-591(50mg/kg) & $19.5(13-27) *$ & $0 *$ \\
XC-591(100mg/kg) & $6(0-8)^{*}$ & $30 *$ \\
\hline
\end{tabular}

*significantly $(\mathrm{P}<0.05)$ differs from the control groups according to Mann-Whitney test.

small molecule targeted drugs, which target key proteins of oncogenic pathways, have shown good effects in the management of many cancers [7-9].

In this study, we attempted to study the biological activities of XC-591, a new benzothiazole-2-thiol derivative, in detail. XC-591 showed obvious antiproliferative activity on many cancer cells by MTT assay.
Compared with other tumor cells, murine mammary tumor cell line 4T1 was more sensitive to XC-591 than others and thus was chosen for further study. XC-591 administered p.o. displayed a marked antitumor activity in 4T1 tumor models. The histological analysis showed XC591 significantly reduced percentages of PCNA-positive nuclei, increased percentages of TUNEL positive nuclei
A

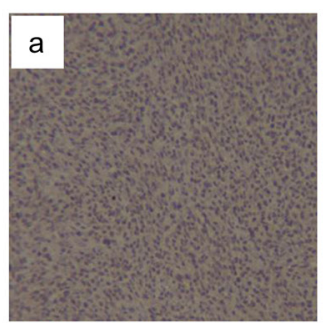

C

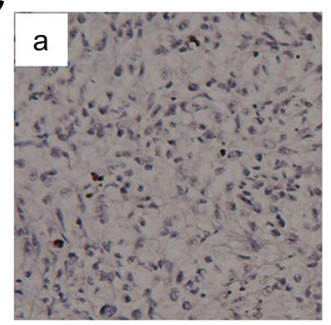

E

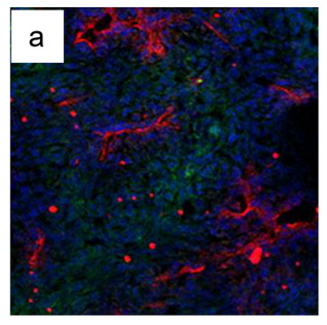

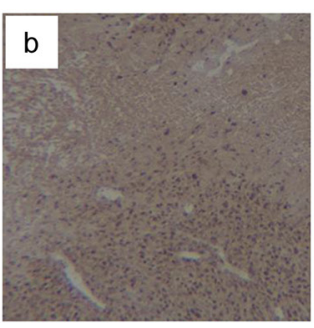
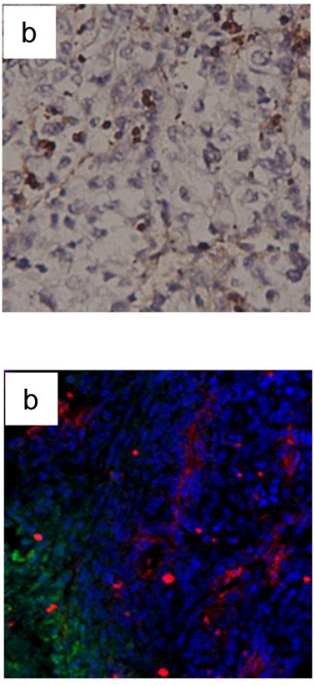

$B$
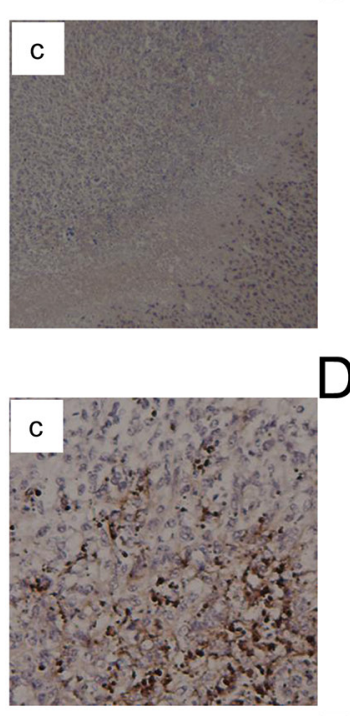

D
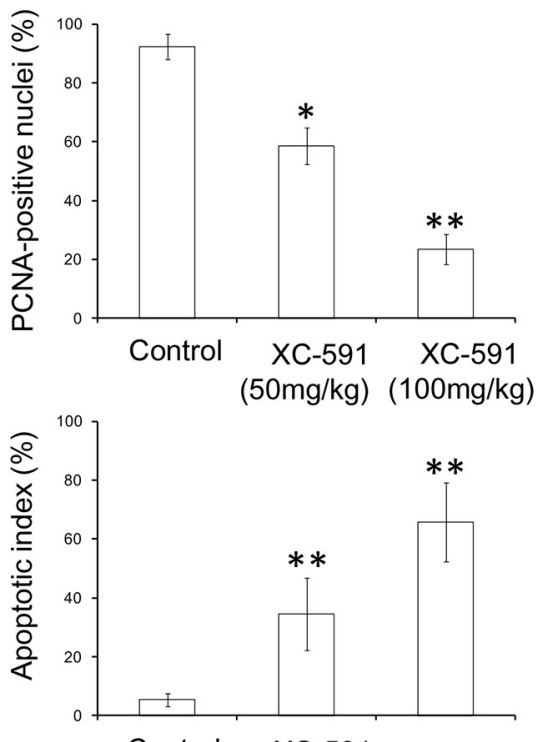

$\mathrm{F}$

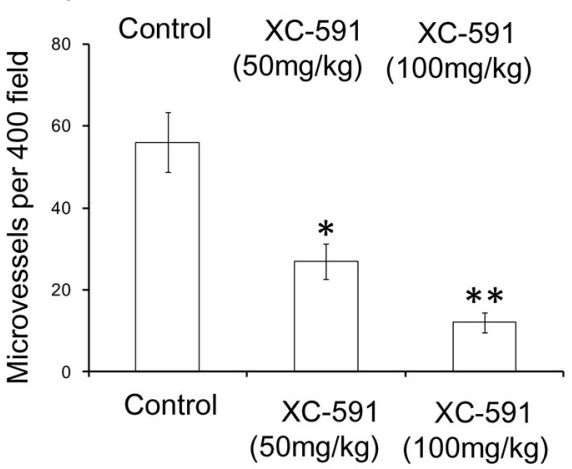

Figure 5: PCNA immunohistochemistry, TUNEL analysis and CD31 staining. A. Representative pictures from PCNA immunohistochemistry. a: untreated group; b: $50 \mathrm{mg} / \mathrm{kg}$ XC-591 treated group; c: $100 \mathrm{mg} / \mathrm{kg}$ XC-591 treated group. B. Quantified values shown were the average percentage of PCNA-positive nuclei. C. Representative pictures from TUNEL. a: untreated group; b: $50 \mathrm{mg} / \mathrm{kg}$ XC-591 treated group; c: $100 \mathrm{mg} / \mathrm{kg}$ XC-591 treated group. D. Percent apoptosis in each group. E. Shown are representative sections from CD31 staining. a: untreated group; b: $50 \mathrm{mg} / \mathrm{kg}$ XC-591 treated group; c: $100 \mathrm{mg} / \mathrm{kg}$ XC-591 treated group. F. Quantified values shown were MVD in each group. * indicates $\mathrm{P}<0.05, * *$ indicates $\mathrm{P}<0.01$. 
and inhibited angiogenesis in a concentration-dependent way. During the whole experiment, no potential toxicity induced by XC-591 treatment was observed. In addition, in order to further study the molecular mechanism, we used western blot to examine the expression level of RhoGDI (predicted drug target protein), cleaved caspase-3 and phosphorylated Akt. The data showed that XC-591 could inhibit RhoGDI, activate caspase- 3 and reduce phosphorylated Akt.

Rho family GTPases work in diverse cellular functions, including morphology, migration, gene transcription and cell cycle [10]. RhoGDI can inhibit nucleotide exchange and membrane association to downregulate activities of Rho family GTPases [11]. RhoGDI is overexpressed in diverse human cancers, including lung cancer, melanoma, ovarian cancer and breast cancer [12-14]. The increased RhoGDI is associated with radiochemotherapy resistance and poor progonosis [15]. For example, a comparative proteomic study showed that RhoGDI was increased in oral squamous cell carcinoma and validated as an independent prognostic factor [16]. Furthermore, RhoGDI was identified as a metastasisassociated protein in colon and prostate cancer [17]. In addition, it has been reported that RhoGDI could stimulate the transcriptional activity of estrogen receptor $\alpha(E R \alpha)$ via a RhoGTPase-dependent pathway that acts on estrogen receptor co-activators GRIP1 and CBP/p300 in breast cancer [18]. RhoGDI1 could increase both liganddependent and -independent ER $\alpha$ activity in breast cancer [19]. Thus, RhoGDI is a very promising anti-cancer target protein in breast cancer.

The important role of RhoGDI in tumor growth is supported by a number of important experimental observations. The suppression of RhoGDI alone by siRNA has a profound inhibitory effect on the growth of hepatocellular carcinoma [20]. Overexpressed RhoGDI can inhibit the induction of apoptosis by cytotoxic drugs in breast cancer cells. Silencing of RhoGDI by siRNA can increase the sensitivity of chemotherapy drugs, such as etoposide, doxorubicin and so on

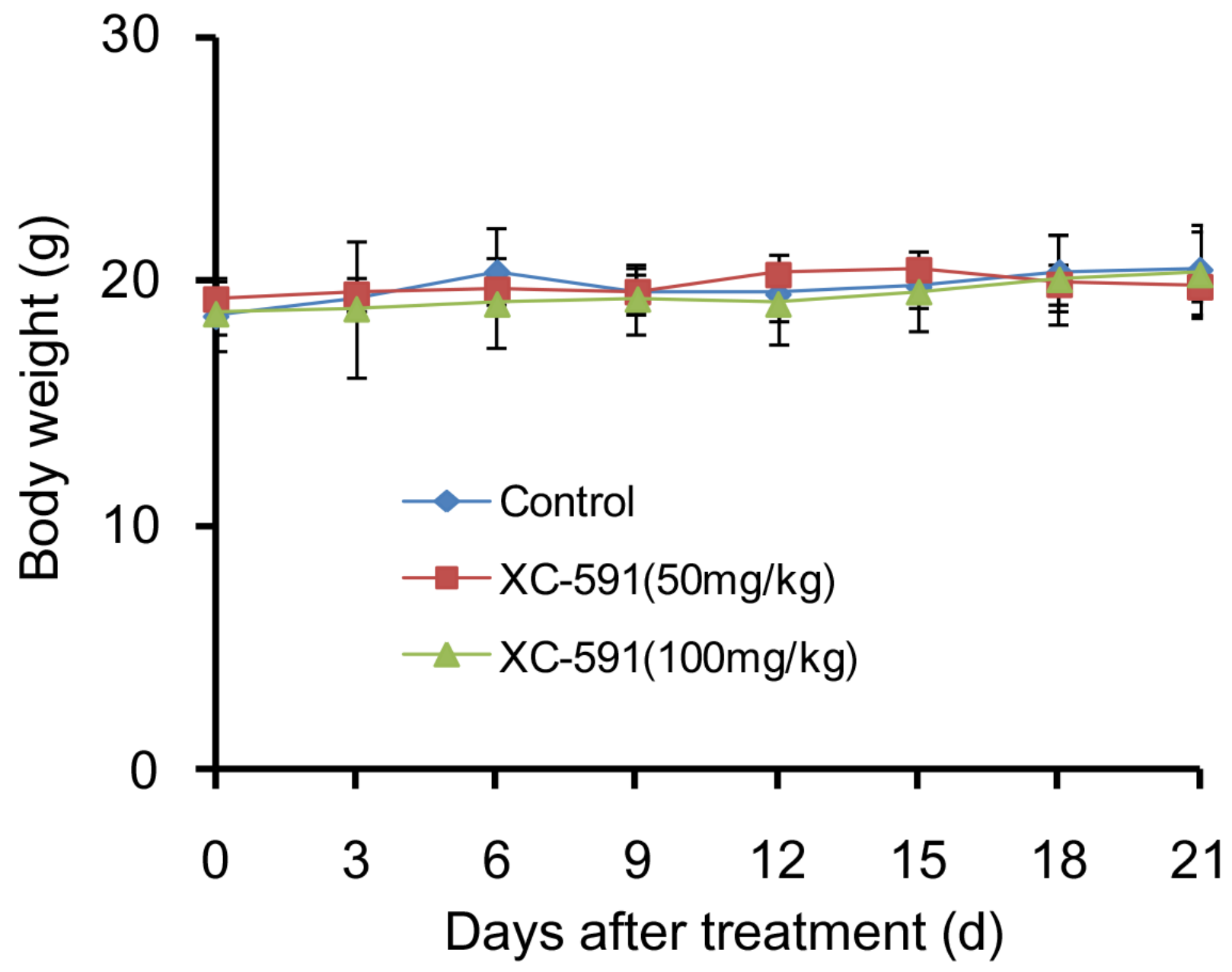

Figure 6: Body weight curves of each group. There were no significant differences in body weight among the three groups (P $>0.05)$. 
Table 3: The blood toxicity of XC-591 treatment

\begin{tabular}{lcccccc}
\hline Treatment & RBC $\left(\times \mathbf{1 0}^{\mathbf{1 2}} / \mathbf{L}\right)$ & HGB $(\mathbf{g} / \mathbf{L})$ & WBC $(\times \mathbf{1 0} / \mathbf{L})$ & PLT $(\times \mathbf{1 0} / \mathbf{L})$ & ALT $(\mathbf{U} / L)$ & AST $(\mathbf{U} / L)$ \\
\hline Control & $7.6 \pm 0.3$ & $138.2 \pm 13.6$ & $7.3 \pm 0.5$ & $543.7 \pm 18.9$ & $32.6 \pm 2.3$ & $98.6 \pm 6.8$ \\
XC-591 $(50 \mathrm{mg} / \mathrm{kg})$ & $7.7 \pm 0.7$ & $136.1 \pm 17.3$ & $7.6 \pm 0.8$ & $523.7 \pm 16.7$ & $36.4 \pm 4.5$ & $96.3 \pm 12.3$ \\
XC-591 $(100 \mathrm{mg} / \mathrm{kg})$ & $7.5 \pm 0.5$ & $141.2 \pm 15.4$ & $7.5 \pm 0.9$ & $537.9 \pm 21.5$ & $35.3 \pm 3.5$ & $96.4 \pm 6.4$ \\
\hline
\end{tabular}

Data are expressed as the mean \pm S.D.

$[21,22]$. These findings mentioned above showed that it is a good method to treat cancer by down-regulating RhoGDI. However, until now, no specific RhoGDI small molecule inhibitors have been designed and studied.

In conclusion, we developed a new benzothiazole2-thiol derivative called XC-591. It showed good anti-cancer activity without obvious toxicity in vitro and in vivo. The molecular mechanism study showed that XC-591 could inhibit its target protein RhoGDI, and thus activated caspase- 3 and decreased phosphorylated Akt. The present data may be useful for further exploration of this new small-molecule inhibitor in the treatment of breast cancer.

\section{XC-591 ( $\mu \mathrm{M})$}

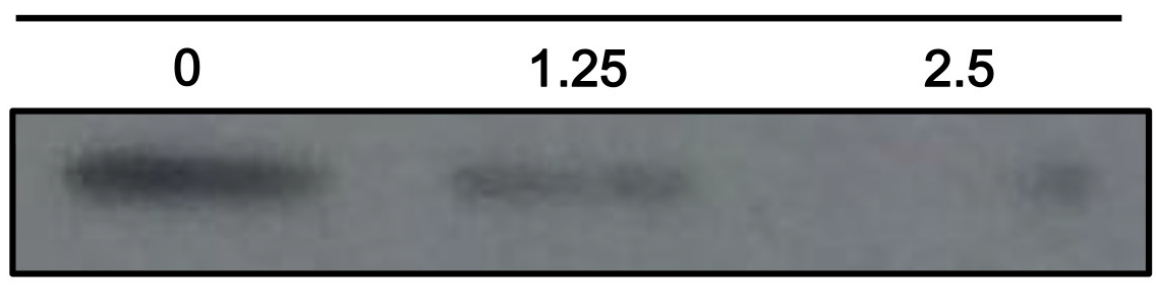

RhoGDI

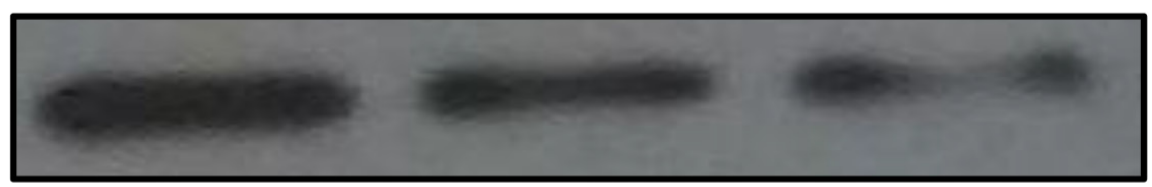

p-AKT

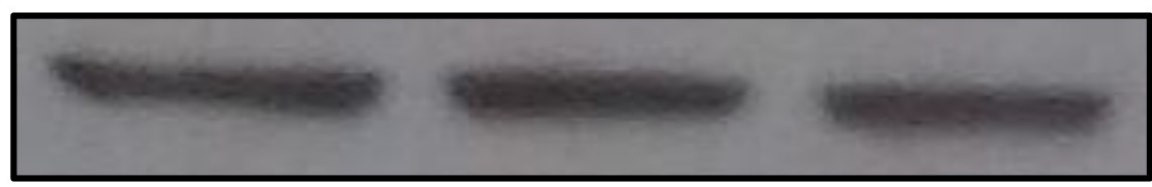

\section{AKT}

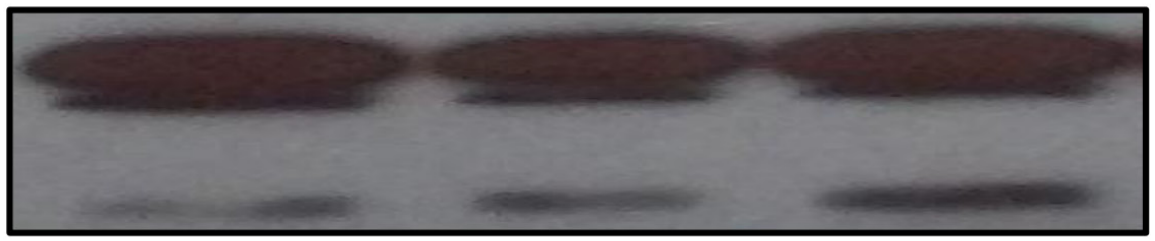

Pro-caspase-3

Cleaved-caspase-3

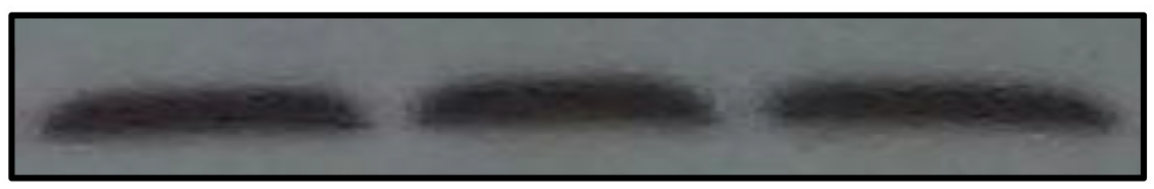

\section{GAPDH}

Figure 7: XC-591 inhibited RhoGDI, activated caspase-3 and reduced phosphorylated Akt. 4T1 cells were treated with XC591 at various concentrations for $48 \mathrm{~h}$ and then were analyzed the expression levels of RhoGDI, p-AKT, AKT, pro-caspase-3 and cleaved caspase-3 by western blot. 


\section{MATERIALS AND METHODS}

\section{Cell culture}

Mouse colon carcinoma cell line CT26, mouse mammary tumor cell line 4T1, mouse Lewis lung carcinoma cell line LLC, mouse melanoma cell line B16, mouse fibrosarcoma cell line MethA, human lung carcinoma cell line A549, human cervical cancer cell line HeLa, human prostate cancer cell line DU145, mouse mammary normal epithelial cell line MM3MG and human normal kidney cell line HK2 were obtained from American Type Culture Collection (ATCC, Manassas, VA, USA). Human normal liver cell line LO2 was purchased from Cell biology of shanghai institute, shanghai, china. They were cultured in DMEM (Life Technologies, Bedford, MA, USA) or RPMI 1640 (Life Technologies).

\section{Synthesis of XC-591}

The route adapted for the synthesis of compound XC-591 was reported before [6].

\section{MTT assay}

Cells were seeded in 96-well plates and cultured for $24 \mathrm{~h}$, followed by XC-591 treatment for $48 \mathrm{~h}$. According to the instructions, MTT was done as reported before [6].

\section{Colony formation assay}

4T1 cells were plated and then treated with $0,1.25$ and $2.5 \mu \mathrm{M}$ of XC-591, respectively. After 7-10 days of incubation, the cells were stained with $0.5 \%$ crystal violet in absolute ethanol and colonies were counted under dissection microscope.

\section{In vivo tumor experiment}

To study the antitumor activities of XC-591 in vivo, 4T1 mice mammary tumor metastatic models were established. In brief, $1 \times 10^{5} 4 \mathrm{~T} 1$ cells were subcutaneously injected into the right dorsal flank of 6 to 8 weeks old female BALB/c mice. The tumor-bearing mice were randomly put into the following three groups (ten mice per treatment group) and each mouse received the corresponding treatment by intragastric administration once daily: (a) control group; (b) XC-591, $50 \mathrm{mg} / \mathrm{kg}$; (c) XC-591, $100 \mathrm{mg} / \mathrm{kg}$. Tumor volumes were evaluated according to the following formula: tumor volume $\left(\mathrm{mm}^{3}\right)=0.52 \times$ length $\times$ width $^{2}$. After sacrificed, tumor net weight was weighed. Autopsy was performed to assess the number and diameter of the metastatic nodules of lung.

\section{Immunohistochemistry}

The primary antibody for PCNA was purchased from Santa Cruz Biotechnology (sc-7907, Santa Cruz,
CA, USA). The primary antibody for CD31 was purchased from Abcam (ab28364, Abcam, Cambridge, United Kingdom). According to instructions of the Envision System-HRP method (DakoCytomation Inc, Carpinteria, CA, USA), tumor sections were stained.

\section{TUNEL assay}

According to the instructions, TUNEL (terminal deoxynucleotidyl transferase mediated dUTP nickend labeling) was completed to assess the percentage of apoptotic cells within tumors. Percent apoptosis was determined by counting the number of apoptotic cells and dividing by the total number of cells in the field ( 5 high power fields/slide).

\section{Toxicity assessment}

To study the potential side effects in the XC-591 treated mice, they were continuously observed for relevant indexes such as weight loss, ruffled fur, diarrhea, anorexia, skin ulcer or toxic deaths. After sacrificed, various organs (lung, liver, kidney, heart, and spleen) were stained with $\mathrm{H} \& \mathrm{E}$, and observed by two pathologists in a blinded manner. The levels of serum ALT and AST were determined with an automatic multifunction-biochemical analyzer. Complete blood counts and differentials were measured within each sample using an Abbott CELL-DYN 3700 hematology analyzer (Abbott Laboratories, Abbott Park, IL, USA).

\section{Western blot}

The western blot was done as previously described [7]. The primary antibodies for RhoGDI and Akt/p-Akt were purchased from Cell Signaling Technology (Beverly, MA, USA). The primary antibodies for caspase-3 and GAPDH were acquired from Santa Cruz Biotechnology.

\section{Statistical analysis}

Statistical analysis of the differences in tumor volume, tumor net weight, animal weight, percentages of apoptosis, percentages of PCNA-positive nuclei and microvessel density were done using one-way analysis of variance (ANOVA). $\mathrm{P}<0.05$ was considered statistically significant. Statistical analysis of the differences in pulmonary metastasis was analyzed using the Mann-Whitney test. P < 0.05 was considered statistically significant.

\section{ACKNOWLEDGMENTS}

The work was supported by National Natural Sciences Foundation of China (81402494 and 81672386) and Science and technology support program of sichuan province (2015SZ0076 and 2014HH0063) and supported by international visiting program for excellent young scholars of SCU. 


\section{CONFLICTS OF INTEREST}

None.

\section{REFERENCES}

1. Chen W, Zheng R, Baade PD, Zhang S, Zeng H, Bray F, Jemal A, Yu XQ and He J. Cancer statistics in China, 2015. CA Cancer J Clin. 2016; 66:115-132.

2. McDonald ES, Clark AS, Tchou J, Zhang P and Freedman GM. Clinical Diagnosis and Management of Breast Cancer. J Nucl Med. 2016; 57:9S-16S.

3. Fouad TM, Kogawa T, Liu DD, Shen Y, Masuda H, El-Zein R, Woodward WA, Chavez-MacGregor M, Alvarez RH, Arun B, Lucci A, Krishnamurthy S, Babiera G, Buchholz TA, Valero V and Ueno NT. Overall survival differences between patients with inflammatory and noninflammatory breast cancer presenting with distant metastasis at diagnosis. Breast Cancer Res Treat. 2015; 152:407-416.

4. Hutchinson I, Chua MS, Browne HL, Trapani V, Bradshaw TD, Westwell AD and Stevens MF. Antitumor benzothiazoles. 14. Synthesis and in vitro biological properties of fluorinated 2-(4-aminophenyl)benzothiazoles. J Med Chem. 2001; 44:1446-1455.

5. Kok SH, Gambari R, Chui CH, Yuen MC, Lin E, Wong RS, Lau FY, Cheng GY, Lam WS, Chan SH, Lam KH, Cheng CH, Lai PB, Yu MW, Cheung F, Tang JC, et al. Synthesis and anti-cancer activity of benzothiazole containing phthalimide on human carcinoma cell lines. Bioorg Med Chem. 2008; 16:3626-3631.

6. Wang Z, Shi XH, Wang J, Zhou T, Xu YZ, Huang TT, Li YF, Zhao YL, Yang L, Yang SY, Yu LT and Wei YQ. Synthesis, structure-activity relationships and preliminary antitumor evaluation of benzothiazole-2-thiol derivatives as novel apoptosis inducers. Bioorg Med Chem Lett. 2011; 21:1097-1101.

7. Peng $X$, Xie G, Wang Z, Lin H, Zhou T, Xiang P, Jiang Y, Yang S, Wei Y, Yu L and Zhao Y. SKLB-163, a new benzothiazole-2-thiol derivative, exhibits potent anticancer activity by affecting RhoGDI/JNK-1 signaling pathway. Cell Death Dis. 2014; 5:e1143.

8. Liu M, Li Z, Yang J, Jiang Y, Chen Z, Ali Z, He N and Wang Z. Cell-specific biomarkers and targeted biopharmaceuticals for breast cancer treatment. Cell Prolif. 2016; 49:409-420.

9. Jiahao Huanga MX, Piyao Gaoc, Yu Yed, Yun Liue, Yang Zhaof, Wenjing Luog, Zhian Lingd, Yunfei Caoa, Sen Zhanga, Feng Gaoa, Weizhong Tang. Antiproliferative effects of formononetin on human colorectal cancer via suppressing cell growth in vitro and in vivo. PROCESS BIOCHEMISTRY. 2015; 50:912-917.
10. Etienne-Manneville S and Hall A. Rho GTPases in cell biology. Nature. 2002; 420:629-635.

11. Dovas A and Couchman JR. RhoGDI: multiple functions in the regulation of Rho family GTPase activities. Biochem J. 2005; 390:1-9.

12. Poland J, Schadendorf D, Lage H, Schnolzer M, Celis JE and Sinha P. Study of therapy resistance in cancer cells with functional proteome analysis. Clin Chem Lab Med. 2002; 40:221-234.

13. Sinha P, Kohl S, Fischer J, Hutter G, Kern M, Kottgen E, Dietel M, Lage H, Schnolzer $M$ and Schadendorf D. Identification of novel proteins associated with the development of chemoresistance in malignant melanoma using two-dimensional electrophoresis. Electrophoresis. 2000; 21:3048-3057.

14. Fritz G, Brachetti C, Bahlmann F, Schmidt M and Kaina B. Rho GTPases in human breast tumours: expression and mutation analyses and correlation with clinical parameters. Br J Cancer. 2002; 87:635-644.

15. Zhao L, Wang H, Li J, Liu Y and Ding Y. Overexpression of Rho GDP-dissociation inhibitor alpha is associated with tumor progression and poor prognosis of colorectal cancer. J Proteome Res. 2008; 7:3994-4003.

16. Chiang WF, Ho HC, Chang HY, Chiu CC, Chen YL, Hour TC, Chuang SJ, Wu YJ, Chen HR, Chen JH, Liu SY, Lu $\mathrm{CL}$ and Chen JY. Overexpression of Rho GDP-dissociation inhibitor alpha predicts poor survival in oral squamous cell carcinoma. Oral Oncol. 2011; 47:452-458.

17. Yamashita $T$, Okamura $T$, Nagano $K$, Imai $S$, Abe $Y$, Nabeshi H, Yoshikawa T, Yoshioka Y, Kamada H, Tsutsumi $\mathrm{Y}$ and Tsunoda S. Rho GDP-dissociation inhibitor alpha is associated with cancer metastasis in colon and prostate cancer. Pharmazie. 2012; 67:253-255.

18. Su LF, Knoblauch R and Garabedian MJ. Rho GTPases as modulators of the estrogen receptor transcriptional response. J Biol Chem. 2001; 276:3231-3237.

19. Su LF, Wang $Z$ and Garabedian MJ. Regulation of GRIP1 and CBP Coactivator activity by Rho GDI modulates estrogen receptor transcriptional enhancement. J Biol Chem. 2002; 277:37037-37044.

20. Wang H, Wang B, Liao Q, An H, Li W, Jin X, Cui S and Zhao L. Overexpression of RhoGDI, a novel predictor of distant metastasis, promotes cell proliferation and migration in hepatocellular carcinoma. FEBS Lett. 2014; 588:503-508.

21. Zhang B, Zhang Y, Dagher MC and Shacter E. Rho GDP dissociation inhibitor protects cancer cells against druginduced apoptosis. Cancer Res. 2005; 65:6054-6062.

22. Storvold GL, Andersen TI, Perou CM and Frengen E. SiRNA: a potential tool for future breast cancer therapy? Crit Rev Oncog. 2006; 12:127-150. 